\title{
Impact of novel processing techniques on the functional properties of egg products and derivatives: a review
}

\begin{abstract}
Eggs are an excellent source of quality proteins. Eggs as a whole and its components (egg white and egg yolk) are employed in a range of food preparations. Thermal processing employed for stabilizing and improving shelf-life of egg components is known to have adverse effect on heatsensitive proteins leading to protein denaturation and aggregation thus, reducing the required functional, technological, and overall quality of egg proteins and other constituents. Therefore, the current challenge is to identify novel processing techniques that not only improve the intrinsic functional properties of eggs or its components, but also improve the quality of the product. This review focuses on the use of technologies such as ultrasound, pulsed electric field, high-pressure processing, radiofrequency, ultraviolet light, microwave, and cold plasma for egg products. These novel technologies are known for their advantages over thermal treatments especially in protecting the heat sensitive nature and retaining the overall quality of the egg and egg products. Availability of alternatives processing has significantly improved the structural properties, techno-functional, nutritional and as well improving the safety egg and egg products. PRACTICAL APPLICATION: Eggs are consumed worldwide as whole egg or in some cases, consumed partly as egg whites or egg yolks. Egg components with improved techno-functional properties can be used in various food industries (such as baking, confectionery, and culinary preparation, etc.). Value addition of new products can be achieved through modification of egg proteins. Additionally, these techniques also provide microbial safety and have a reduced impact on nutritional content and overall food quality.
\end{abstract}

